\title{
Visfatin concentration is decreased in women with gestational diabetes mellitus in the third trimester
}

\author{
M. Akturk1, A.E. Altinova2, I. Mert³, U. Buyukkagnici4, A. Sargin33, M. Arslan'1, and N. Danisman ${ }^{3}$ \\ 1Department of Endocrinology and Metabolism, Faculty of Medicine, Gazi University; 2Division of Endocrinology; ${ }^{3}$ Department of \\ Obstetrics and Gynecology; 4Division of Biochemistry, Zekai Tahir Burak Women's Health Education and Research Hospital, \\ Ankara, Turkey
}

\begin{abstract}
Our aim is to investigate visfatin concentration and its relationship to glycated hemoglobin $\left(\mathrm{HbA}_{1 \mathrm{c}}\right)$, insulin resistance, lipid parameters, and neonatal birth weight in women with gestational diabetes mellitus (GDM). In our study group, there were 47 women with GDM and 31 women with normal glucose tolerance (NGT) between 33-39 weeks of gestation. Plasma visfatin levels were significantly decreased in pregnant women with GDM compared to those with NGT $(p=0.001)$. Homeostasis model assessmentinsulin resistance (HOMA-IR) levels were higher in the GDM group than in the NGT group $(p=0.006)$. In all subjects, plasma visfatin levels were negatively correlated with HOMA-IR, post-prandial blood glucose, triglycerides, and VLDL cholesterol $(p<0.05)$. We did not observe any statistically signifi-
\end{abstract}

\section{INTRODUCTION}

Visfatin, initially considered a pre-B cell colony enhancing factor, is a novel adipocytokine and is preferentially produced by visceral fat $(1,2)$. Visfatin may have a substantial role in the glucose homeostasis $(1,3)$. Visfatin concentrations have been reported to be affected by glucose and insulin in humans (3). There are conflicting studies on visfatin concentrations in Type 2 diabetes mellitus (DM). Increased as well as decreased circulating visfatin has been observed in patients with Type 2 DM (4-6). Therefore, the exact role of visfatin in Type 2 DM is not fully known.

Insulin sensitivity decreases by $30-60 \%$ in normal pregnancy when compared to healthy non-pregnant women (7). Gestational diabetes mellitus (GDM) is observed in almost $7 \%$ of all pregnancies (8). GDM is characterized by glucose intolerance, emerging first in pregnancy (9). Insulin resistance seems to be more apparent with increasing gestational weeks (7). Women with GDM are at high risk of subsequently developing Type 2 DM (10). Data available on visfatin levels in patients with GDM are also controversial. To date, two previous studies $(11,12)$ reported increased visfatin concentrations in GDM, whereas others $(13,14)$ showed that GDM is associated with decreased visfatin concentrations.

Since visfatin is an adipocytokine that is associated with glucose homeostasis, we hypothesized that maternal visfatin concentrations might be altered in pregnant women with GDM in the third trimester, which is an advanced state of insulin resistance. Therefore, in the current study,

Key-words: Birth weight, gestational diabetes mellitus, HOMA-IR, third trimester, visfatin. Correspondence: M. Akturk, Bilkent University, Merkez Loj. 21/5, Bilkent, Ankara, Turkey, 06800

E-mail:mujdeakturk@hotmail.com

Accepted September 24, 2007. cant correlation between the plasma visfatin levels and the selected parameters in the GDM group, but in the NGT group plasma visfatin levels were negatively correlated with HOMA-IR ( $r=-0.36, p=0.04)$. There was no correlation between visfatin concentrations and fetal birth weight in either group $(p>0.05)$. By regression analysis, having GDM was found to be the only significant determinant $(t=3.5$, $p=0.001)$ of visfatin concentration ( $\left.R=0.39, r^{2}=0.15\right)$. We conclude that women with GDM have significantly decreased visfatin concentrations in the third trimester. Future studies are required to establish the exact role of visfatin in the pathogenesis of GDM.

(J. Endocrinol. Invest. 31: 610-613, 2008)

@2008, Editrice Kurtis

our main aim is to investigate visfatin levels and their possible relationship with insulin resistance, lipid parameters, glycated hemoglobin $\left(\mathrm{HbA}_{1 \mathrm{c}}\right)$, and neonatal birth weight in women with GDM in comparison to women with normal glucose tolerance (NGT).

\section{MATERIALS AND METHODS}

The study was performed with 47 women with GDM [mean age; $30.1 \pm 0.6 \mathrm{yr}$, body mass index (BMI); $\left.29.2 \pm 0.5 \mathrm{~kg} / \mathrm{m}^{2}\right]$ and 31 women with NGT (mean age; $28.5 \pm 0.7 \mathrm{yr}, \mathrm{BMl} ; 28.0 \pm 0.6 \mathrm{~kg} / \mathrm{m}^{2}$ ) between 33-39 weeks of gestation on follow-up at the Department of Obstetrics and Gynecology and the Department of Endocrinology. Screening of a 50-g oral glucose challenge test (GCT) was performed for all subjects. Women with blood glucose levels above $140 \mathrm{mg} / \mathrm{dl}$ at the screening test underwent an oral glucose tolerance test (OGTT). GDM was diagnosed when subjects had two or more abnormal values for the OGTT (normal values are fasting $<95,1-\mathrm{h}<180,2-\mathrm{h}<155$, and $3-\mathrm{h}<140$ $\mathrm{mg} / \mathrm{dl}$ ) between $24-28$ weeks according to the diagnostic criteria of American Diabetes Association (ADA) (8). The NGT group was also evaluated with regard to fasting blood glucose (FBG) levels (below $95 \mathrm{mg} / \mathrm{dl}$ ) and 1-h post-prandial blood glucose (PPBG) levels (below $140 \mathrm{mg} / \mathrm{dl}$ ). The exclusion criteria were defined as any systemic disease known to affect inflammatory markers, recent infection, high blood pressure and associated disease (preeclampsia and eclampsia), pregnancies of assisted reproductive techniques, recent betamethasone use, chronic drug use (except for vitamins and iron), or multiple pregnancies. None of the pregnant women were in active labor. Estimation of gestational age was based on routine ultrasonographic screening. Informed consent was obtained from all participants and the local Ethics Committee approved the study.

Blood samples were collected after an 8-h overnight fast, and were immediately separated and stored at $-80 \mathrm{C}$ until analysis. Visfatin, FBG, PPBG, insulin, and lipid parameters were deter- 
mined in all subjects between 33-39 weeks. $\mathrm{HbA}_{1 c}$ was measured in subjects with GDM. Serum $\mathrm{HbA}_{1 c}$ levels were measured by turbidimetric inhibition immunoassay (Roche Diagnostics $\mathrm{GmbH}$, Mannheim, Germany). Glucose levels were measured with standard enzymatic methods (Roche Diagnostic GmbH, Manheim, Germany). Serum concentrations of total cholesterol, HDL cholesterol (HDL-C), and triglycerides (TG) were measured using an enzymatic calorimetric method with commercially available kits (Roche Diagnostic $\mathrm{GmbH}$, Manheim, Germany). VLDL cholesterol (VLDL-C) levels were calculated as TG/5. LDL cholesterol levels were calculated using the Friedewald equation. Plasma visfatin levels were determined with enzyme immunoassay [Visfatin C-Terminal (Human) EIA, Phoenix Pharmaceuticals, Inc, Germany]. The intra-assay and inter-assay coefficients of variations were below $5 \%$ and $14 \%$, respectively. Minimum detectable concentration of visfatin was $2.84 \mathrm{ng} / \mathrm{ml}$. Serum insulin levels were measured by immunoradiometric assay (sandwich type assay) using an insulin IRMA kit (Immunotech, Prague, Czech Republic). The intra-assay and interassay coefficients of variation for insulin were $4.3 \%$ and $3.4 \%$, respectively. The minimum detectable concentration of insulin was $0.5 \mu \mathrm{lU} / \mathrm{ml}$. Insulin resistance was estimated by using the homeostasis model assessment-insulin resistance (HOMA-IR) index, calculated as [serum glucose level $(\mathrm{mmol} / \mathrm{l}) \mathrm{x}$ insulin $(\mu \mathrm{lU} / \mathrm{ml})] / 22.5$ (15).

Since only 65 out of 78 women ( 40 women with GDM and 25 women with NGT) delivered their babies in the studied hospital, we could only reach the hospital records for these neonates. Thus, we evaluated and compared plasma visfatin levels with respect to the birth week and birth weight in both study groups. Statistical analysis of the study was performed using SPSS version 11.5 for Windows software (Statistical Package for Social Science, Chicago, IL, USA). Data were expressed as the mean \pm SEM. To determine whether or not differences existed between the groups, a t-test or Mann-Whitney $U$ test was used. $p$-values below 0.05 were considered statistically significant. For univariate correlation between continuous variables, Spearman coefficients were used. In multivariate analysis, we utilized a stepwise regression analysis. Non-normally distributed data were logarithmically transformed before analysis.

\section{RESULTS}

No statistically significant differences were found between the GDM group and NGT group with respect to age, and BMI $(p>0.05)$ in the third trimester. The clinical and biochemical characteristics of subjects are shown in Table 1. HOMA-IR levels were higher in the GDM group $(4.9 \pm 0.8)$ than in the NGT group $(3.6 \pm 0.9)(p=0.006)$. Plasma visfatin levels were significantly decreased in women with GDM compared to those of women with NGT $(75.3 \pm 4.9$ vs $110.8 \pm 7.8 \mathrm{ng} / \mathrm{ml}, \mathrm{p}=0.001)$.

In Table 2, we reported the results of Spearman's correlation test between the plasma visfatin levels with the selected parameters. Plasma visfatin levels were negatively correlated with HOMA-IR, PPBG, TG, and VLDL-C in all subjects $(p<0.05)$. We did not observe any statistically significant correlation between the plasma visfatin levels with the selected parameters (such as gestational week, BMI, FBG, and others) in the GDM group ( $p>0.05$ ) as shown in Table 2. But in the NGT group, plasma visfatin levels were negatively correlated with HOMA-IR $(r=-0.36, p=0.04)$.
By regression analysis, a model consisting of visfatin as a dependent variable and GDM/NGT, log HOMA-IR, PP$B G, \log T G$, and $\log V L D L-C$ as independent variables was constructed. Based on this, GDM was found to be a significant determinant $(t=3.5, p=0.001)$ of visfatin concentrations $\left(R=0.39, r^{2}=0.15\right)$.

Birth weeks and birth weights were comparable between the GDM group (no. $=40)(38.9 \pm 0.2$ week, $3379 \pm 83 \mathrm{~g})$ and the NGT group (no.=25) $(39.3 \pm 0.3$ week, $3276 \pm 74 \mathrm{~g})$ ( $p=0.3$ and $p=0.4$, respectively). There was no correlation between maternal visfatin concentrations and fetal birth weight in either group $(p>0.05)$. One in the GDM group and 2 in the NGT group delivered at preterm. When these cases were excluded from the statistical analysis, there were no significant differences with respect to birth week and birth weight in either group. Furthermore, our findings on correlation analyses between birth weight and plasma visfatin levels still remained statistically insignificant in both groups, and in the entire population as well. Six (12.8\%) in the GDM group were treated with insulin in addition to nutritional therapy and 41 patients (87.2\%) in the GDM group were treated with nutritional therapy alone. There was no significant difference in visfatin levels between women treated with only nutritional therapy $(73.3 \pm 5.2 \mathrm{ng} / \mathrm{ml})$ or nutritional plus insulin therapy $(88.8 \pm 14.8 \mathrm{ng} / \mathrm{ml})(p=0.2)$.

\section{DISCUSSION}

The current study shows that plasma visfatin concentrations were markedly decreased in GDM compared with NGT in the third trimester of gestation. Contrary to our findings, Lewandowski et al. (12) found an increase in visfatin levels in 16 patients with GDM at 28 weeks of gestation. They also reported increased visfatin concentrations with worsening of glucose tolerance. Similarly,

Table 1 - The characteristics of pregnant woman with gestational diabetes mellitus (GDM) and normal glucose tolerance (NGT).

\begin{tabular}{lccc}
\hline & with GDM & with NGT & $p$ \\
\hline No. & 47 & 31 & \\
Age $(\mathrm{yr})$ & $30.1 \pm 0.6$ & $28.5 \pm 0.7$ & 0.08 \\
BMI $\left(\mathrm{kg} / \mathrm{m}^{2}\right)$ & $29.2 \pm 0.5$ & $28.0 \pm 0.6$ & 0.2 \\
Preconceptional & $24.9 \pm 0.7$ & $23.8 \pm 0.8$ & 0.3 \\
BMI (kg/m²) & & & \\
HOMA-lR & $4.9 \pm 0.8$ & $3.6 \pm 0.9$ & $0.006^{*}$ \\
FBG (mg/dl) & $81.6 \pm 2.9$ & $75.1 \pm 1.4$ & $0.2^{*}$ \\
PPBG (mg/dl) & $126.7 \pm 4.5$ & $99.8 \pm 3.1$ & $<0.001^{*}$ \\
HbA $1 \mathrm{c}(\%)$ & $5.5 \pm 0.1$ & & \\
TC (mg/dl) & $248.0 \pm 4.8$ & $251.4 \pm 9.4$ & $0.9^{*}$ \\
LDL-C (mg/dl) & $121.4 \pm 6.3$ & $130.8 \pm 8.6$ & $0.4^{*}$ \\
VLDL-C (mg/dl) & $55.2 \pm 4.0$ & $46.7 \pm 2.6$ & $0.1^{*}$ \\
HDL-C(mg/dl) & $72.8 \pm 2.5$ & $73.8 \pm 3.2$ & $0.8^{*}$ \\
TG (mg/dl) & $276.1 \pm 20.2$ & $234.0 \pm 13.4$ & $0.1^{*}$ \\
Visfatin (ng/ml) & $75.3 \pm 4.9$ & $110.8 \pm 7.8$ & $0.001^{*}$ \\
\hline Da are expressed & &
\end{tabular}

Data are expressed as means \pm SEM, $p>0.05$ statistically non-significant, *with Mann Whitney U test. BMI: body mass index; HOMA-IR: homeostasis model assessment-insulin resistance; FBG: fasting blood glucose PPBG: post-prandial blood glucose; $\mathrm{HbA}_{1}$ : glycated hemoglobin; TC: total cholesterol; LDL-C: LDL cholesterol; VLDL-C: VLDL cholesterol; HDLC: HDL cholesterol; TG: triglycerides. 
Krzyzanowska et al. (11) investigated visfatin concentrations in women with GDM between 28-30 and 38-40 weeks of gestation and after delivery. Authors reported that women with GDM had increased concentrations of visfatin compared to healthy pregnant controls. On the other hand, studies by Chan et al. (13) and Haider et al. (14) revealed decreased visfatin concentrations in patients with GDM. Chan et al. (13) measured visfatin levels in 20 women with GDM at 24 to 28 weeks of gestation and found that plasma visfatin concentrations were significantly lower in GDM than those in healthy pregnant women. In agreement with this study, Haider et al. (14) reported recently that women with GDM at 24-28 weeks of gestation had low fasting plasma visfatin and suppressed visfatin response when compared to women with NGT during OGTT. The results on this particular study are somehow conflicting. The underlying reason may be associated with the largely unknown metabolism of visfatin. Besides, which factors exactly influence circulating visfatin concentrations in GDM have not been established yet. Nevertheless, present data regarding visfatin levels in Type 2 DM and obesity are also controversial. Previous studies $(4,5)$ reported increased circulating visfatin in patients with Type 2 DM, whereas another study (6) found that Type 2 diabetic patients showed decreased plasma visfatin concentrations. Circulating visfatin levels are reduced after weight loss in obese subjects (16), yet, in contrast, another study (17) found that weight loss has an increasing effect on visfatin concentrations.

As far as we know, there is no published study with respect to the changes in placental visfatin expression and the relationship of visfatin to insulin resistance in GDM. A possible reason for decreased maternal visfatin in women with GDM in our study might be associated with some unknown factors that can lower the secretion of visfatin from placenta and fetal membranes of diabetic pregnant

Table 2 - Spearman's correlation coefficients of plasma visfatin levels with selected variables in pregnant woman with gestational diabetes mellitus (GDM) and normal glucose tolerance (NGT).

\begin{tabular}{|c|c|c|c|c|c|c|}
\hline & \multicolumn{2}{|c|}{ All groups } & \multicolumn{2}{|c|}{ with GDM } & \multicolumn{2}{|c|}{ with NGT } \\
\hline & $\mathrm{r}$ & $p$ & $\mathrm{r}$ & $p$ & $\mathrm{r}$ & $p$ \\
\hline Age (yr) & -0.11 & 0.3 & -0.13 & 0.3 & 0.08 & 0.6 \\
\hline Gestational week & 0.16 & 0.1 & 0.07 & 0.6 & -0.008 & 0.9 \\
\hline BMI (kg/m²) & 0.03 & 0.7 & 0.07 & 0.6 & 0.21 & 0.2 \\
\hline $\begin{array}{l}\text { Preconceptional } \\
\mathrm{BMI}\left(\mathrm{kg} / \mathrm{m}^{2}\right)\end{array}$ & 0.01 & 0.9 & 0.1 & 0.4 & -0.04 & 0.9 \\
\hline HOMA-IR & -0.30 & 0.008 & -0.1 & 0.4 & -0.36 & 0.04 \\
\hline FBG (mg/dl) & -0.20 & 0.07 & -0.2 & 0.16 & -0.10 & 0.5 \\
\hline PPBG (mg/dl) & -0.27 & 0.01 & -0.1 & 0.37 & 0.09 & 0.5 \\
\hline $\mathrm{HbA}_{1 \mathrm{c}}(\%)$ & & & -0.1 & 0.38 & & \\
\hline $\mathrm{TC}$ (mg/dl) & -0.09 & 0.4 & -0.1 & 0.3 & -0.04 & 0.7 \\
\hline LDL-C (mg/dl) & 0.06 & 0.5 & 0.07 & 0.6 & -0.03 & 0.8 \\
\hline VLDL-C (mg/dl) & -0.23 & 0.03 & -0.2 & 0.08 & -0.02 & 0.8 \\
\hline $\mathrm{HDL}-\mathrm{C}(\mathrm{mg} / \mathrm{dl})$ & 0.10 & 0.3 & 0.1 & 0.3 & 0.005 & 0.9 \\
\hline TG (mg/dl) & -0.22 & 0.04 & -0.2 & 0.09 & -0.01 & 0.9 \\
\hline Birth weight (g) & -0.15 & 0.2 & -0.02 & 0.8 & -0.30 & 0.1 \\
\hline
\end{tabular}

BMI: body mass index; HOMA-IR: homeostasis assessment-insulin resistance; FBG: fasting blood glucose; PPBG: post-prandial blood glucose; $\mathrm{HbA}_{1 \mathrm{c}}$ : glycated hemoglobin; TC: total cholesterol; LDL-C: LDL cholesterol; VLDL-C: VLDL cholesterol; HDL-C: HDL cholesterol; TG: triglycerides. women. For example, placental visfatin expression in normal pregnancy has been shown in a previous study (18). Malamitsi-Puchner et al. (19) suggested that placental expression and transfer of visfatin could be responsible for intrauterine visfatin concentrations in normal full-term pregnancies, as well. The correlation between maternal and fetal visfatin levels in intrauterine growth-restricted and normal pregnancies was found in a different study by the same group (20). This supports the idea of transplacental transport of visfatin. Therefore, placental visfatin expression should be investigated in GDM.

Furthermore, visfatin concentrations might be under the effects of some hormones (including human placental lactogen and progesterone) that circulate in high concentrations during pregnancy (21), although the effect of these hormones on circulating visfatin in GDM is not currently known. On the other hand, it may be speculated that insulin therapy could effect our results since insulin administration was shown to decrease visfatin concentrations (14). However, this effect is unlikely because our patients were mainly treated with nutritional therapy alone. Another key point may be related with the changes in visceral fat tissue in GDM since plasma visfatin levels were found to be increased in proportion to visceral fat accumulation (2). Changes in regional fat distribution and increased intra-abdominal visceral fat accumulation have been reported during normal pregnancy (22). Post-partum visceral fat was found to be greater in patients with previous GDM who also had current impaired glucose tolerance (23). However, to the best of our knowledge, there is not enough evidence about changes in visceral fat composition in pregnant women with GDM. If there is an increase in visceral fat accumulation in patients with GDM, decreased visfatin may be a defensive mechanism in these patients. Taken together, further studies are needed to reveal the unknown mechanisms associated with alterations in visfatin concentrations in patients with GDM.

In the current study, although visfatin levels have been negatively correlated with insulin resistance in all subjects, we observed that there was a significant negative correlation between visfatin and HOMA-IR in the NGT group, whereas a similar correlation did not exist in the GDM group. Pregnancy is a state that creates variable degrees of insulin resistance and our results suggest that visfatin is associated with the degree of insulin resistance in normal pregnant women, but not in women with GDM. In a study performed in non-diabetic pregnant women, maternal visfatin levels in the first trimester were found to predict second trimester insulin sensitivity (24). On the other hand, some previous studies reported that pregnant women with intrauterine growth retardation had increased maternal visfatin levels compared to controls $(20,25)$. Post-natal first day fetal fasting insulin was observed to be decreased in the study by Malamitsi-Puchner et al. (20). Interestingly, our results showing that women with GDM had decreased visfatin levels suggest this kind of relationship between fetal insulin level and maternal visfatin since fetuses from women with GDM were expected to be hyperinsulinemic. However, we could not measure fetal visfatin and insulin levels in our study. Therefore, the association of maternal visfatin with the degree of fetal insulin sensitivity or resistance deserves further investigation. 
Visfatin concentrations have been reported to be negatively correlated with HOMA-IR and TG in a study covering the relatives of the patients with familial hyperlipidemia and non-diabetic subjects (26). In the current study, we also found that plasma visfatin levels were negatively correlated with TG and VLDL-C in all subjects. Yet, we did not observe such a relationship in a multiple regression analysis, except for GDM.

Previous studies revealed that maternal adipocytokines have considerable effects on neonatal birth weight (27). A positive relationship between neonatal visfatin and birth weight was shown in normal pregnancies $(19,28)$. In the current study, we found no association between third trimester maternal visfatin concentrations and birth weight.

The results of the present study indicate that women with GDM had significantly decreased visfatin concentrations in the third trimester. Furthermore, maternal visfatin levels at the third trimester are not a determinant of fetal birth weight. Future studies are required to establish the exact role of visfatin in the pathogenesis of GDM.

\section{REFERENCES}

1. Pilz S, Mangge H, Obermayer-Pietsch B, März W. Visfatin/pre-Bcell colony-enhancing factor: a protein with various suggested functions. J Endocrinol Invest 2007, 30: 138-44.

2. Sandeep S, Velmurugan K, Deepa R, Mohan V. Serum visfatin in relation to visceral fat, obesity, and type 2 diabetes mellitus in Asian Indians. Metabolism 2007, 56: 565-70.

3. Haider DG, Schaller G, Kapiotis S, Maier C, Luger A, Wolzt M. The release of the adipocytokine visfatin is regulated by glucose and insulin. Diabetologia 2006, 49: 1909-14.

4. Chen MP, Chung FM, Chang DM, et al. Elevated plasma level of visfatin/pre-B cell colony-enhancing factor in patients with type 2 diabetes mellitus. J Clin Endocrinol Metab 2006, 91: 295-9.

5. López-Bermejo A, Chico-Julià B, Fernàndez-Balsells $M$, et al. Serum visfatin increases with progressive beta-cell deterioration. Diabetes 2006, 55: 2871-5.

6. Li L, Yang G, Li Q, et al. Changes and relations of circulating visfatin, apelin, and resistin levels in normal, impaired glucose tolerance, and type 2 diabetic subjects. Exp Clin Endocrinol Diabetes 2006, 114: 544-8.

7. Kautzky-Willer A, Bancher-Todesca D. Endocrine changes in diabetic pregnancy. In: Djelmiš J, Desoye G, Ivanišević M eds. Diabetology of pregnancy. Frontiers in diabetes. Vol. 17. Basel: Karger 2005, 18-33.

8. American Diabetes Association. Gestational diabetes mellitus. Diabetes Care 2004, 27 (suppl 1): S88-90.

9. Buchanan TA, Xiang AH. Gestational diabetes mellitus. J Clin Invest 2005, 115: 485-91.

10. Kim C, Newton KM, Knopp RH. Gestational diabetes and the incidence of type 2 diabetes: a systematic review. Diabetes Care 2002, 25: $1862-8$.
11. Krzyzanowska K, Krugluger W, Mittermayer F, et al. Increased visfatin concentrations in women with gestational diabetes mellitus. Clin Sci (Lond) 2006, 110: 605-9.

12. Lewandowski KC, Stojanovic N, Press M, et al. Elevated serum levels of visfatin in gestational diabetes: a comparative study across various degrees of glucose tolerance. Diabetologia 2007, 50: 1033-7.

13. Chan TF, Chen $\mathrm{YL}$, Lee $\mathrm{CH}$, et al. Decreased plasma visfatin concentrations in women with gestational diabetes mellitus. J Soc Gynecol Investig 2006, 13: 364-7.

14. Haider DG, Handisurya A, Storka A, et al. Visfatin response to glucose is reduced in women with gestational diabetes mellitus. Diabetes Care 2007, 30: 1889-91.

15. Matthews DR, Hosker JP, Rudenski AS, Naylor BA, Treacher DF, Turner RC. Homeostasis model assessment: insulin resistance and beta-cell function from fasting plasma glucose and insulin concentrations in man. Diabetologia 1985, 28: 412-9.

16. Haider DG, Schindler K, Schaller G, Prager G, Wolzt M, Ludvik B. Increased plasma visfatin concentrations in morbidly obese subjects are reduced after gastric banding. J Clin Endocrinol Metab 2006, 91: 1578-81.

17. Krzyzanowska K, Mittermayer F, Krugluger W, Kopp HP, Schernthaner G. Increase in visfatin after weight loss induced by gastroplastic surgery. Obesity (Silver Spring) 2006, 14: 1886-9.

18. Ognjanovic S, Bryant-Greenwood GD. Pre-B-cell colony-enhancing factor, a novel cytokine of human fetal membranes. Am J Obstet Gynecol 2002, 187: 1051-8.

19. Malamitsi-Puchner A, Briana DD, Gourgiotis D, Boutsikou M, Baka $\mathrm{S}$, Hassiakos D. Blood visfatin concentrations in normal full-term pregnancies. Acta Paediatr 2007, 96: 526-9.

20. Malamitsi-Puchner A, Briana DD, Boutsikou M, Kouskouni E, Hassiakos D, Gourgiotis D. Perinatal circulating visfatin levels in intrauterine growth restriction. Pediatrics 2007,119: e1314-8.

21. Kuhl C. Etiology and pathogenesis of gestational diabetes. Diabetes Care 1998, 21 (Suppl 2): B19-26.

22. Kinoshita $\mathrm{T}$, Itoh M. Longitudinal variance of fat mass deposition during pregnancy evaluated by ultrasonography: the ratio of visceral fat to subcutaneous fat in the abdomen. Gynecol Obstet Invest 2006, 61: 115-8.

23. Lim S, Choi SH, Park YJ, et al. Visceral fatness and insulin sensitivity in women with a previous history of gestational diabetes mellitus. Diabetes Care 2007, 30: 348-53.

24. Mastorakos G, Valsamakis G, Papatheodorou DC, et al. The role of adipocytokines in insulin resistance in normal pregnancy: visfatin concentrations in early pregnancy predict insulin sensitivity. Clin Chem 2007, 53: 1477-83.

25. Fasshauer M, Blüher M, Stumvoll M, Tönessen P, Faber R, Stepan $H$. Differential regulation of visfatin and adiponectin in pregnancies with normal and abnormal placental function. Clin Endocrinol (Oxf) 2007, 66: 434-9.

26. Wang $P$, van Greevenbroek MM, Bouwman FG, et al. The circulating PBEF/NAMPT/visfatin level is associated with a beneficial blood lipid profile. Pflugers Arch 2007, 454: 971-6.

27. López-Bermejo A, Fernández-Real JM, Garrido E, et al. Maternal soluble tumour necrosis factor receptor type 2 (sTNFR2) and adiponectin are both related to blood pressure during gestation and infant's birthweight. Clin Endocrinol (Oxf) 2004, 61: 544-52.

28. Briana DD, Boutsikou M, Gourgiotis D, et al. Role of visfatin, insulin-like growth factor-I and insulin in fetal growth. J Perinat Med 2007, 35: 326-9. 